\title{
Natural Killer Cells in Healthy and Diseased Subjects
}

\author{
Roberto Biassoni, ${ }^{1}$ John E. Coligan, ${ }^{2}$ and Lorenzo Moretta ${ }^{1}$ \\ ${ }^{1}$ Molecular Medicine, Department of Experimental Medicine, Instituto Giannina Gaslini, Genova 16147, Italy \\ ${ }^{2}$ Receptor Cell Biology Section, Laboratory of Immunogenetics, NIAID, NIH, Rockville, MD 20852, USA
}

Correspondence should be addressed to Roberto Biassoni, robertobiassoni@ospedale-gaslini.ge.it

Received 19 June 2011; Accepted 19 June 2011

Copyright ( $\odot 2011$ Roberto Biassoni et al. This is an open access article distributed under the Creative Commons Attribution License, which permits unrestricted use, distribution, and reproduction in any medium, provided the original work is properly cited.

Natural killer cells or NK cells have been known since the 70 s on the basis of their functional properties $[1,2]$, but essentially no molecular markers existed able to specifically identify them. Still in the early eighties, the few scientists that worked in NK cells field had difficulty convincing about the existence of those cells while, in the same period, immunologists were essentially in the search of the $\delta$-chain of the T-cell receptor.

Nowadays, following the molecular characterization of different surface receptors and the signalling pathways as well as the characterization of the molecules specifically recognized and the analysis of their 3D-structures, the research on NK cells has become a highly competitive field. Researchers studying different functional and molecular aspects have been challenged to understand the function of these cells and the mechanism of the regulation of their function in terms of NK cell licensing or education and NK memory. In addition, it is always more complex the crosstalk with other cell of the immune system either by cell contact or through the release of soluble factors. Briefly, natural killer cells are responsible for the immune responses against tumor or virally infected cells. Their function is tightly regulated by a clonal and stochastic distribution of germline-encoded cell surface receptors, and these molecules are able to deliver either inhibitory or activating signals. Thus, every NK cell is equipped by at least a single inhibitory receptor which is functionally dominant and that senses the level of surface expression of MHC class I molecules on autologous cells mediating self-tolerance [3-15]. This function has been maintained during mammalian evolution by expansion of different multigene families coding for receptors with marked structural divergences that have been evolved in the different species together with appropriate MHC molecules serving as ligand [16]. Any alteration on target cell of the surface expression level of the MHC class I molecules, induced by viral infection or tumor transformation, induces NK-mediated cell killing. NK cell receptors evolved in a highly dynamic fashion, primarily driven by the necessity to deal with a large variety of pathogens and to recognize properties of cells characteristic of tumor transformation.

In this special issue, a series of reviews or articles on natural killer cells are published and summarize information related to NK cells and diseases and the rationale use of these knowledge for clinical applications. In detail we summarize the molecular and structural analysis of NK receptor and their interaction with ligands and how the diversity of KIR genotype and of HLA class I molecules are related with the outcome of a number of key human infections $[17,18]$. Other contributions are based on the analysis of the knowledges regarding the molecular interactions between NK cells and myeloid antigen-presenting cells and their role in the regulation/polarization of adaptive immune responses [19, 20]. The ability of distinct species of gut-derived commensal bacterial to differently affect the outcome of DC/NK crosstalk and the Th1 polarization of the adaptive immune response is also discussed $[19,20]$. The complex network of interactions involving the immune system is also through the use of cytokines. The functional redundancy and the specific role of the common gamma-chain cytokine family (IL-2, IL-4, IL7, IL-9, IL-15, and IL-21) in the regulation of the immune response and in the homeostasis of the lymphoid cells and the acquisition of memory-like functions have been analyzed [21]. In addition, novel strategies to deliver cytokines and the use of immunokine aimed to maximize their therapeutic 
potential and to costimulate the NK cell activation by enhancing NK cell adhesion to target cells have been described $[21,22]$. Several reviews analyze the mechanism of recognition used by NK cells to sense viral pathogens, like HCV, HBV, HIV, CMV, and other infectious diseases [1,23-25] and the potential role of NK cells during severe sepsis and septic shock [26]. Cytomegaloviruses evolved different mechanisms to hide and survive to immune-related responses. Innate immunity is the first line of defence in the case of CMV infections, and viral particles are known to modulate the expression of ligand molecules specific for NK-triggering receptor on the surface of infected cells. In addition, some viral genes are also known to encode MHC class I-like surface molecules contributing to immunoevasion both in mice and in humans $[23,24]$. Since CMVs and their respective hosts coevolved, it is tempting to speculate that the viral MHC class-I-like molecules originate from the host genomes. Revilleza et al. on the bases of nucleotides, proteins, and crystallographic/modelling structural data obtained from human and mice speculate that a single ancient event of partial genome transfer and not multiple phenomena may have evolved the different CMV class I-like molecules [23]. In various mice strains the capture of host-genome has originally allowed the development of MHC class-I-like molecules able to interact with inhibitory Ly49 receptors. Instead B6 and $\mathrm{Ma} / \mathrm{My}$ have evolved activating Ly 49 molecules, which through the interaction with viral class I-like molecules and/ or with the presence of additional factors are able to trigger NK cell cytolysis rather than inhibit and probably to contribute to the development of NK cell memory [24]. Thus it appears clear that there is a strict interplay among NK cells, their receptors and the responses toward viral pathogens. To address these issues, we have an depth analysis discussing the different triggering receptors expressed by NK cells, their level of expression and the possible ligands involved in the interaction with cells infected by viruses belonging to different families (Orthomyxo-, Paramyxo-, Flavi-, Lentiviridae) [25]. Thanks to the latest review in which we have found discussed the NK-mediated responses following viral pathogen infection, here we find some recent points of view about how also microbial sepsis and the "systemic inflammatory response syndrome" or peptic shock may be related with NK cell-immune responses [26]. All the information regarding NK receptors and their ligands, as well as the fine regulation of innate/acquired immune-responses orchestrated by NK cells and the cytokines network are the basis for better use in cancer therapy. The NK cell responses toward tumor using specific mAbs and an ADCC mechanism and all attempts to increase anti-tumor cell cytotoxicity using cytokine combinations, TLR agonist and immunokines are also discussed $[27,28]$. ADCC is often impaired in PBMC from patients with advanced cancer as a result of NK cell dysfunction probably generated by tumor-produced soluble factor(s) (i.e., TGF $\beta$ ), thus any approaches aimed to increase the ADCC killing are necessary to obtain an efficient tumor cell readication.

Along this line, IL15, IL2 and the use of Lenalidomide, an analog of thalidomide known to increase Fc $\gamma$ R-mediated signalling, adoptive cell transfer and ex-vivo cell expansion, have been successfully used [28]. Although, the firsts attempts in the use of autologous ex vivo expanded cells did not give encouraging results, it is now clear the basis of these failures, making allogeneic cell transfer more attractive. The use of KIR/HLA class I-mismatched cells, selected in order to be not sensitive to inhibitory signals from the recipient HLA ligands, have been proved to be protective against disease relapses [28].

The other face of the coin of NK cells and tumor is shown by Schmitt et al, that focalizes our attention on a rare form of hematolymphoid tumors including the extranodal NK/T-cell lymphoma (NKTCL) that is considered inside the provisional group of chronic NK-cell lymphoproliferative disorder. This lymphoma is apparently associated with EBVassociated malignancy with very poor prognosis, rare in western countries, but it represents at least $10 \%$ of nonHodgkin's lymphomas in Asia and Central/South America [29]. Finally, two reviews are taking care of using the combined analysis of genetic data regarding KIR and HLA class I molecules and their association in autoimmune diseases and the cytokines network below the pathogenesis of chronic inflammation [30,31]. In both cases, a decrease of the strong inhibitory pathways controlled by HLA-C-specific receptors and thus a reduced activating threshold promote immune responses, and this effect of changing the threshold of immune-suppression/activation may contribute to explain the genetic susceptibility of different autoimmune diseases.

In the last years, we have assisted to an explosion in the research field centered on NK cells. In the near future, we will see the publication of different results in the regulation of receptors and ligands expression by miRNA, as exemplified by the recent published article regarding the control of HLA$\mathrm{C}$ expression in HIV infection [32]. There are different arguments that need a detailed study. Among these, we also know that KIR recognizes HLA class I ligands on the basis of the presence of particular amino acid residues; probably different exceptions exist. In addition, very little information is available about the role of viral peptides, presented by HLA class I able to trigger the activation of Killer Ig-like Receptors (KIR2DS, KIR3DS). The absence of such information might explain the failure, so far, of an experimental identification of ligands specific for different triggering KIR, with a single exception [33]. Finally, a complete characterization of the molecules recognized by all the cloned NK cells receptors is still needed to analyze in detail all the triggering pathways induced by NK cell-mediated recognition.

\section{Roberto Biassoni John E. Coligan Lorenzo Moretta}

\section{Acknowledgments}

We would like to thank Irene Vanni and Elisabetta Ugolotti for suggestions about the paper. 


\section{References}

[1] R. B. Herberman, M. E. Nunn, H. T. Holden, and D. H. Lavrin, "Natural cytotoxic reactivity of mouse lymphoid cells against syngeneic and allogeneic tumors. II. Characterization of effector cells," International Journal of Cancer, vol. 16, no. 2, pp. 230-239, 1975.

[2] R. Kiessling, E. Klein, and H. Wigzell, “"Natural” killer cells in the mouse. I. Cytotoxic cells with specificity for mouse Moloney leukemia cells. Specificity and distribution according to genotype," European Journal of Immunology, vol. 5, no. 2, pp. 112-117, 1975.

[3] E. Ciccone, O. Viale, D. Pende et al., "Specific lysis of allogeneic cells after activation of CD3- lymphocytes in mixed lymphocyte culture," Journal of Experimental Medicine, vol. 168, no. 6, pp. 2403-2408, 1988.

[4] H. G. Ljunggren and K. Karre, "In search of the 'missing self': MHC molecules and NK cell recognition," Immunology Today, vol. 11, no. 7, pp. 237-244, 1990.

[5] L. Moretta, E. Ciccone, A. Moretta, P. Hoglund, C. Ohlén, and K. Karre, "Allorecognition by NK cells: nonself or no self?" Immunology Today, vol. 13, pp. 300-306, 1992.

[6] W. M. Yokoyama and W. E. Seaman, "The Ly-49 and NKR-P1 gene families encoding lectin-like receptors on natural killer cells: the NK gene complex," Annual Review of Immunology, vol. 11, pp. 613-635, 1993.

[7] N. Wagtmann, R. Biassoni, C. Cantoni et al., "Molecular clones of the p58 NK cell receptor reveal immunoglobulinrelated molecules with diversity in both the extra- and intracellular domains," Immunity, vol. 2, no. 5, pp. 439-449, 1995.

[8] A. D’Andrea, C. Chang, K. Franz-Bacon, T. McClanahan, J. H. Phillips, and L. L. Lanier, "Molecular cloning of NKB1 a natural killer cell receptor for HLA-B allotypes," Journal of Immunology, vol. 155, no. 5, pp. 2306-2310, 1995.

[9] M. Colonna and J. Samaridis, "Cloning of immunoglobulinsuperfamily members associated with HLA-C and HLA-B recognition by human natural killer cells," Science, vol. 268, no. 5209, pp. 405-408, 1995.

[10] A. Moretta, C. Bottino, M. Vitale et al., "Receptors for HLA class-I molecules in human natural killer cells," Annual Review of Immunology, vol. 14, pp. 619-648, 1996.

[11] E. O. Long and N. Wagtmann, "Natural killer cell receptors," Current Opinion in Immunology, vol. 9, no. 3, pp. 344-350, 1997.

[12] P. E. Posch, F. Borrego, A. G. Brooks, and J. E. Coligan, "HLA-E is the ligand for the natural killer cell CD94/NKG2 receptors," Journal of Biomedical Science, vol. 5, no. 5, pp. 321-331, 1998.

[13] R. Biassoni, "Human natural killer receptors, co-receptors, and their ligands," Current Protocols in Immunology, no. 84, pp. 14.10.1-14.10.40, 2009.

[14] M. Carretero, C. Cantoni, T. Bellon et al., "The CD94 and NKG2-A C-typelectinscovalently assemble to form a natural killer cellinhibitoryreceptor for HLA class I molecules," European Journal of Immunology, vol. 27, no. 2, pp. 563-567, 1997.

[15] F. Borrego, M. Ulbrecht, E. H. Weiss, J. E. Coligan, and A. G. Brooks, "Recognition of human histocompatibility leukocyte antigen (HLA)-E complexed with HLA class I signal sequencederived peptides by CD94/NKG2 confers protection from natural killer cell-mediated lysis," Journal of Experimental Medicine, vol. 187, no. 5, pp. 813-818, 1998.

[16] R. Biassoni, E. Ugolotti, and A. de Maria, "Comparative analysis of NK-cell receptor expression and function across primate species: perspective on antiviral defenses," Self Non Self, vol. 1, no. 2, pp. 103-113, 2010.
[17] K. M. Jamil and S. I. Khakoo, "KIR/HLA interactions and pathogen immunity," Journal of Biomedicine and Biotechnology, vol. 2011, Article ID 298348, 9 pages, 2011.

[18] M. G. Joyce and P. D. Sun, "The structural basis of ligand recognition by natural killer cell receptors," Journal of Biomedicine and Biotechnology, vol. 2011, Article ID 203628, 15 pages, 2011.

[19] O. Chijioke and C. Münz, "Interactions of human myeloid cells with natural killer cell subsets in vitro and in vivo," Journal of Biomedicine and Biotechnology, vol. 2011, Article ID 251679, 7 pages, 2011.

[20] V. Rizzello, I. Bonaccorsi, M. L. Dongarr, L. N. Fink, and G. Ferlazzo, "Role of natural killer and dendritic cell crosstalk in immunomodulation by commensal bacteria probiotics," Journal of Biomedicine and Biotechnology, vol. 2011, Article ID 473097, 10 pages, 2011.

[21] R. Meazza, B. Azzarone, A. M. Orengo, and S. Ferrini, "Role of common-gamma chain cytokines in NK cell development and function: perspectives for immunotherapy," Journal of Biomedicine and Biotechnology, vol. 2011, Article ID 861920, 16 pages, 2011.

[22] L. Kühne, M. Konstandin, Y. Samstag, S. Meuer, T. Giese, and C. Watzl, "WF10 stimulates NK cell cytotoxicity by increasing LFA-1-mediated adhesion to tumor cells," Journal of Biomedicine and Biotechnology, vol. 2011, Article ID 436587, 6 pages, 2011.

[23] M. R. Revilleza, R. Wang, J. Mans, M. Hong, K. Natarajan, and D. H. Margulies, "How the virus outsmarts the host: function and structure of cytomegalovirus MHC-I-like molecules in the evasion Of natural killer cell surveillance," Journal of Biomedicine and Biotechnology, vol. 2011, Article ID 724607, 12 pages, 2011.

[24] M. Pyzik, E.-M. Gendron-Pontbriand, and S. M. Vidal, "The impact of Ly49-NK cell-dependent recognition of MCMV infection on innate and adaptive immune responses," Journal of Biomedicine and Biotechnology, vol. 2011, Article ID 641702, 9 pages, 2011.

[25] F. Marras, F. Bozzano, and A. De Maria, "Involvement of activating NK cell receptors and their modulation in pathogen immunity," Journal of Biomedicine and Biotechnology, vol. 2011, 2011.

[26] L. Chiche, J. -M. Forel, G. Thomas et al., "The role of natural killer cells in sepsis," Journal of Biomedicine and Biotechnology, vol. 2011, Article ID 986491, 8 pages, 2011.

[27] K. L. Alderson and P. M. Sondel, "Clinical cancer therapy by NK cells via antibody-dependent cell mediated cytotoxicity," Journal of Biomedicine and Biotechnology, vol. 2011, Article ID 379123, 7 pages, 2011.

[28] E. M. Levy, M. P. Roberti, and J. Mordoh, "Natural killer cells in human cancer: from biological functions to clinical applications," Journal of Biomedicine and Biotechnology, vol. 2011, Article ID 676198, 11 pages, 2011.

[29] C. Schmitt, N. Sako, M. Bagot, Y. Huang, P. Gaulard, and A. Bensussan, "Extranodal NK/T-cell lymphoma: toward the identification of clinical molecular targets," Journal of Biomedicine and Biotechnology, vol. 2011, Article ID 790871, 11 pages, 2011.

[30] P. K. Yadav, C. Chen, and Z. Liu, "Potential role of NK cells in the pathogenesis of inflammatory bowel disease," Journal of Biomedicine and Biotechnology, vol. 2011, Article ID 348530, 6 pages, 2011.

[31] S. Dunphy and C. M. Gardiner, "NK cells and psoriasis," Journal of Biomedicine and Biotechnology, vol. 2011, Article ID 248317, 10 pages, 2011. 
[32] S. Kulkarni, R. Savan, Y. Qi et al., "Differential microRNA regulation of HLA-C expression and its association with HIV control," Nature, vol. 472, no. 7344, pp. 495-498, 2011.

[33] R. Biassoni, A. Pessino, A. Malaspina et al., "Role of amino acid position 70 in the binding affinity of p50.1 and p58.1 receptors for HLA-Cw4 molecules," European Journal of Immunology, vol. 27, no. 12, pp. 3095-3099, 1997. 


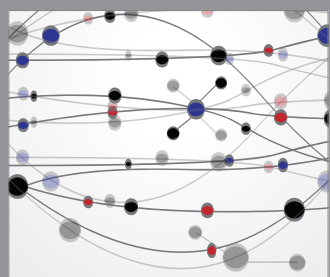

The Scientific World Journal
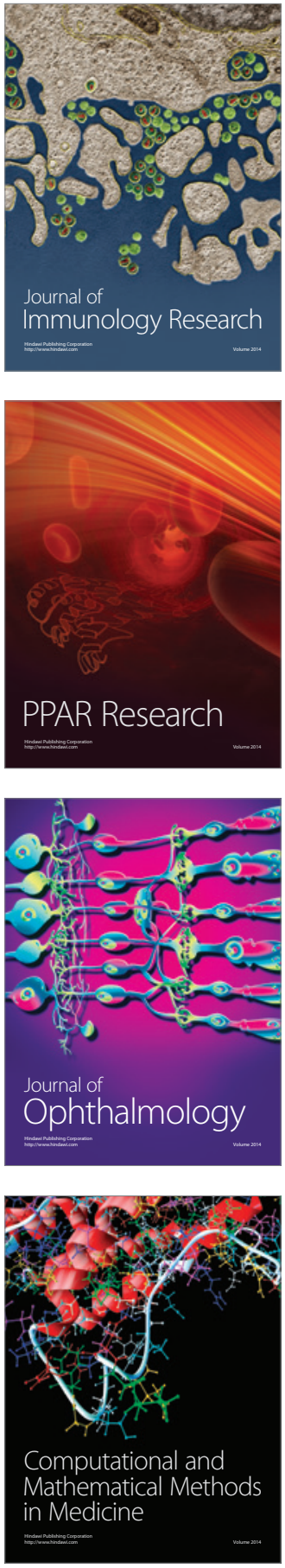

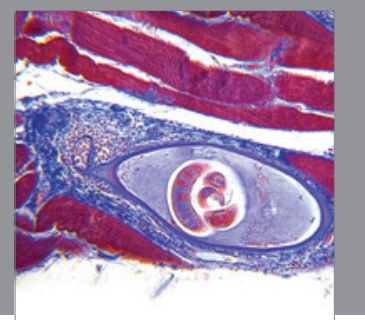

Gastroenterology

Research and Practice
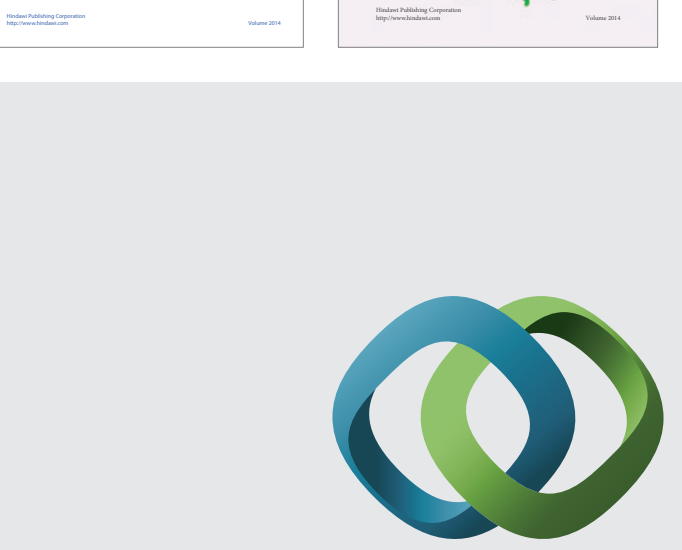

\section{Hindawi}

Submit your manuscripts at

http://www.hindawi.com
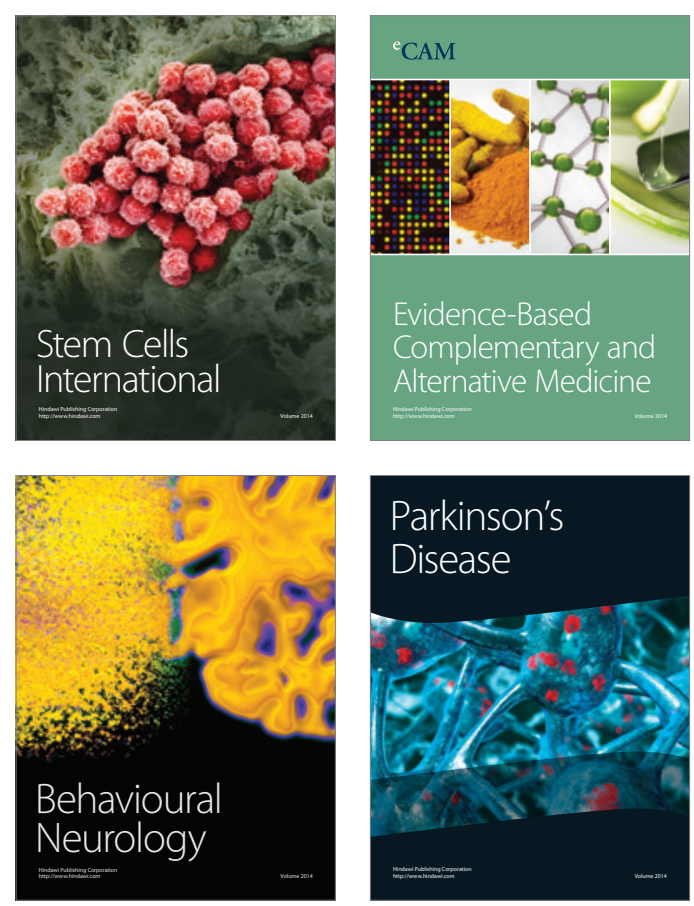

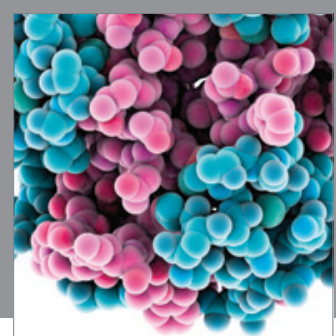

Journal of
Diabetes Research

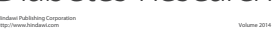

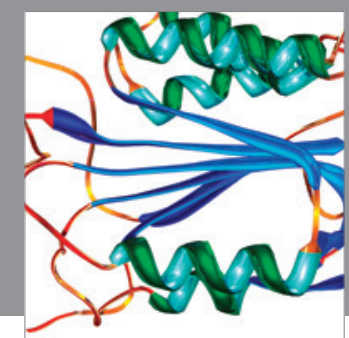

Disease Markers
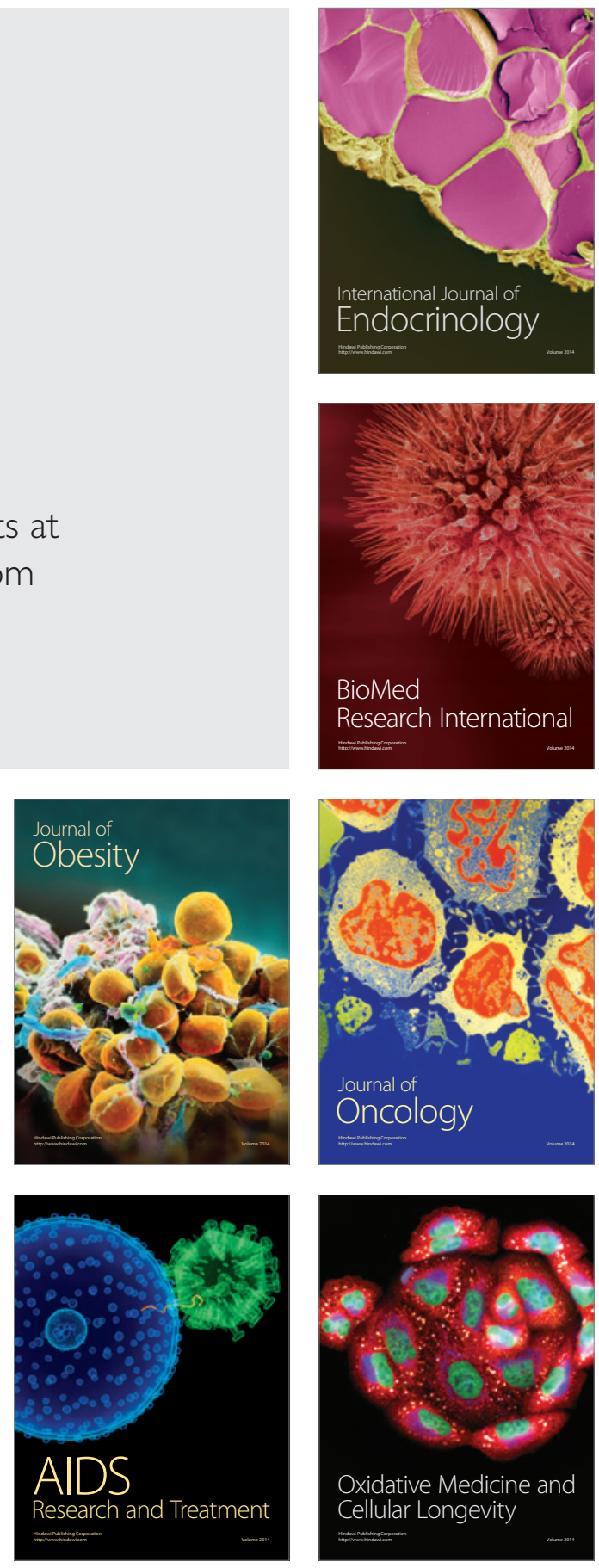